\title{
Reacciones cutáneas severas a medicamentos DRESS y sulfasalazina
}

\author{
Severe drug-induced skin reactions \\ DRESS and sulfasalazine
}

\author{
Adulkarin Patiño, Tatiana Echeverry, Giovanni de la Cruz, \\ Nelcy Teresa Guerra, Lina María Murcia, Carlos Aldana, \\ Alberto de Zubiría • Bogotá, D.C. (Colombia)
}

\begin{abstract}
Resumen
Las reacciones cutáneas severas son una de las manifestaciones adversas de los medicamentos que llegan a poner en peligro la vida. El objetivo del presente artículo es presentar el caso de una paciente con este tipo de reacciones atendido en el Hospital Universitario de La Samaritana. Se trata de una mujer con síntomas cutáneos en quien se documenta reacción severa medicamentosa por sulfasalazina asociado a compromiso hepático y gastrointestinal que responde a la terapia con esteroides y la suspensión de sulfazalazina. (Acta Med Colomb 2012; 37: 83-88).
\end{abstract}

Palabras clave: cutánea, DRESS, medicamento, teacción, dulfasalazina.

\section{Abstract}

Severe skin reactions are one of the adverse drug events that can threaten life. The aim of this paper is to present the case of a female patient seen at the Hospital Universitario La Samaritana, who had this type of reaction. This is a woman with skin symptoms in whom we documented a severe drug-induced skin reaction by sulfasalazine associated to hepatic and gastrointestinal involvement that responded to steroids and the suspension of sulfasalazine. (Acta Med Colomb 2012; 37: 83-88).

Keywords: skin, DRESS, drug, reaction, sulfasalazine.
Dres. Adulkarin Patiño, Tatiana Echeverry, Giovanni de la Cruz, Nelcy Teresa Guerra Malaver y Lina María Murcia Pinilla: Residentes de Medicina Interna, Universidad de la Sabana-Hospital de La Samaritana; Dr. Carlos Aldana: Médico Interno, Hospital Samaritana; Dr. Alberto de Zubiría: Médico Internista-Inmunología Clínica Alérgica, Jefe Departamento de Medicina Interna-Hospital de La Samaritana. Bogotá, D.C. (Colombia).

Financiación. Ninguna.

Conflicto de intereses. No se declaran conflictos de intereses por los autores.

Correspondencia. Dr. Adulkarin Patiño. Universidad de la Sabana-Hospital de La Samaritana. Bogotá, D.C. (Colombia).

E-mail: adulkarin@hotmail.com

Recibido: 26/X/2011 Aceptado: 16/V/2012

\section{Presentación del caso}

Paciente femenino de 56 años de edad, natural y procedente de Cota (Cundinamarca), casada, de profesión hogar. Consulta por cuadro clínico de ocho días de evolución caracterizado por lesiones eritematosas pruriginosas en tórax y abdomen asociado a malestar general y deposiciones diarreicas mucoides. Fue manejada inicialmente con loratadina, caladryl ${ }^{\oplus}$, ranitidina y emoliente. Tiene antecedente de artritis reumatoidea diagnosticada hace tres años en manejo con prednisolona $10 \mathrm{mg} / \mathrm{día}$, metotrexate $7,5 \mathrm{mg} / \mathrm{día}$, carbonato de calcio $600 \mathrm{mg} / \mathrm{día}$, omeprazol $20 \mathrm{mg} /$ día, naproxeno $250 \mathrm{mg} /$ día y sulfasalazina $500 \mathrm{mg} /$ día; este último iniciado dos semanas previas al de inicio de sintomatología.

Al examen físico presentaba una tensión arterial de 130/70 $\mathrm{mmHg}$, una frecuencia cardiaca de 120 latidos por minuto, una frecuencia respiratoria de 18 por minuto, un peso de $106 \mathrm{Kg}$, una talla de $168 \mathrm{~cm}$ y una temperatura de $36^{\circ} \mathrm{C}$. En la piel tenía múltiples lesiones eritematodescamativas con estigmas de rascado en cara, tórax, abdomen y miembros inferiores, con compromiso de palmas y plantas (Fotos A-C). El examen cardiorrespiratorio, gastrointestinal, osteoarticular y neurológico fue normal. En el hemograma se encontró eosinofilia (Tabla

Tabla 1. Hemograma

\begin{tabular}{|l|c|c|c|c|c|c|c|}
\hline & Fecha & $\begin{array}{c}\text { Leucocitos } \\
\mathbf{m i l e s} / \mathbf{m m}^{3}\end{array}$ & $\begin{array}{c}\text { Neutrófilos } \\
(\boldsymbol{\%})\end{array}$ & $\begin{array}{c}\text { Linfocitos } \\
(\%)\end{array}$ & $\begin{array}{c}\text { Eosinófilos } \\
(\%)\end{array}$ & $\begin{array}{c}\text { Plaquetas } \\
\text { miles/mm }\end{array}$ & $\begin{array}{c}\text { HCTO } \\
(\boldsymbol{\%})\end{array}$ \\
\hline Diciembre & $02 / 09$ & 14.070 & 34 & 45 & 12 & 143.000 \\
Diciembre & $03 / 09$ & 11.720 & 78 & 23 & 9 & 368.000 \\
\hline
\end{tabular}




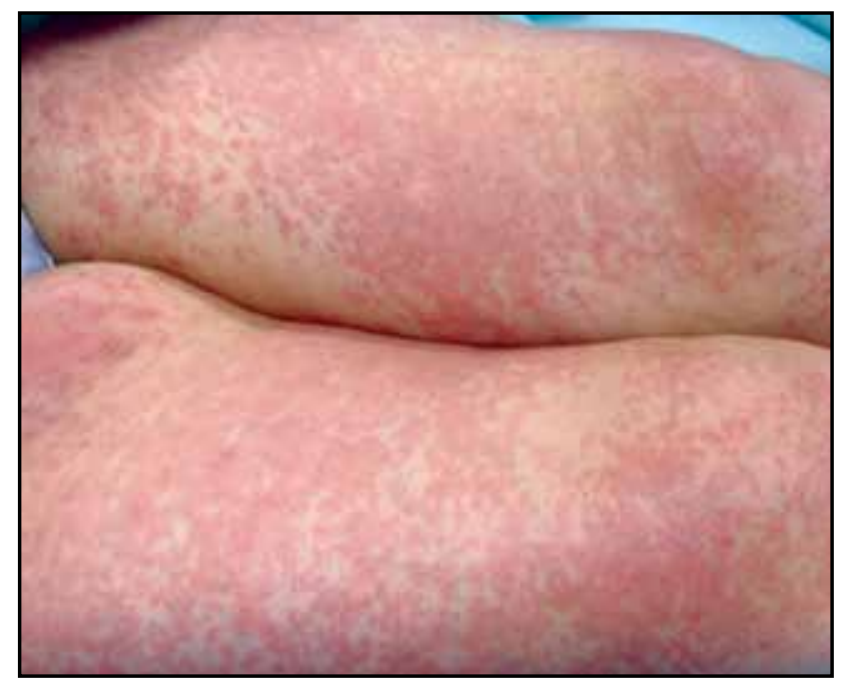

Figura 1A.

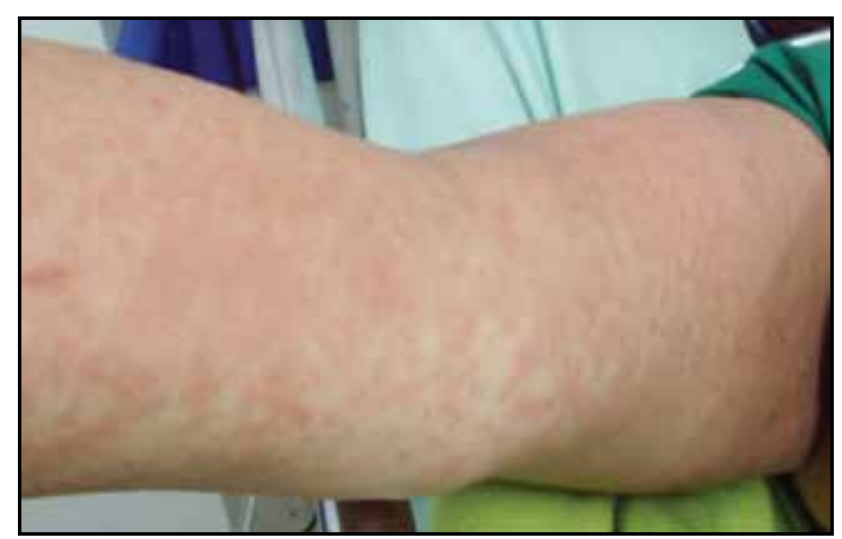

Figura 1C.

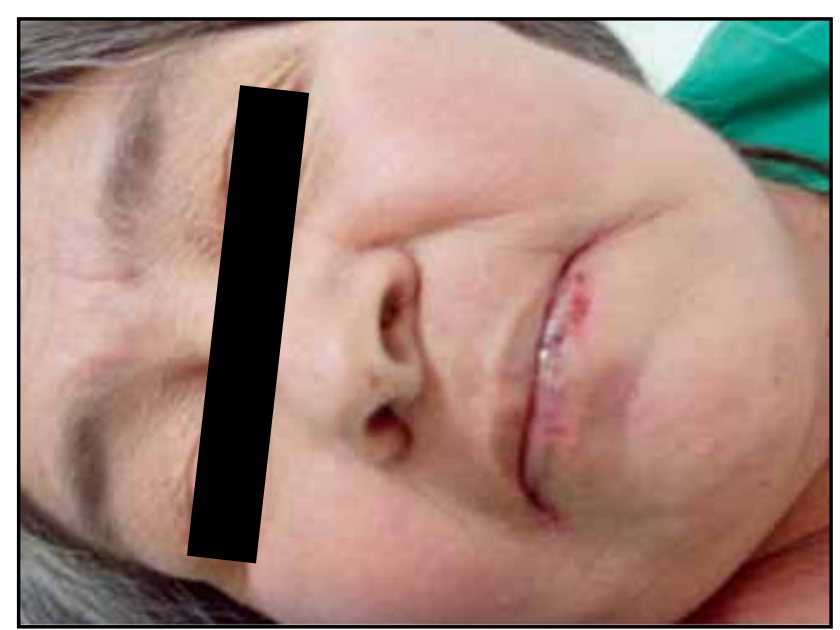

Figura 1E.

Figura 1. DRESS por sulfasalazina. A) Miembros inferiores. B) Abdomen. C) Miembro superior derecho. D) Rostro al ingreso. E) Secuelas de herpes labial.

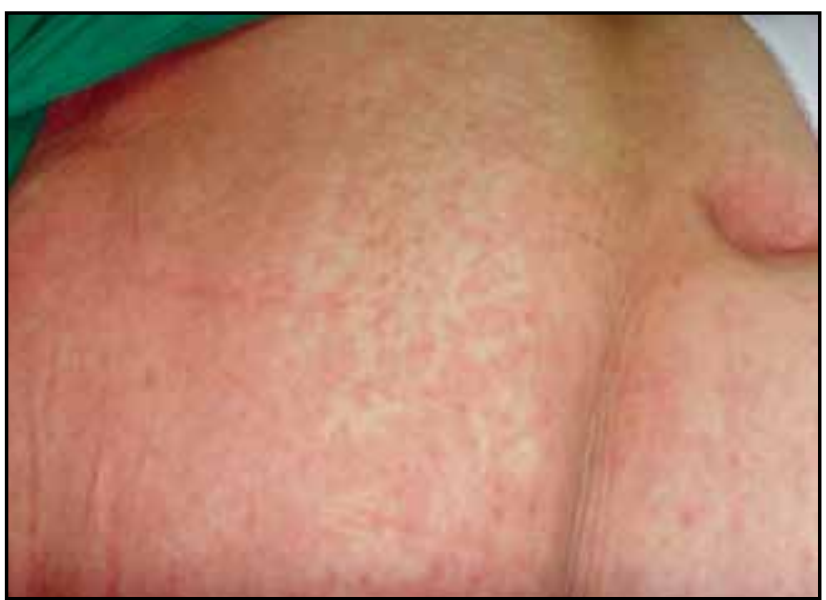

Figura 1B.

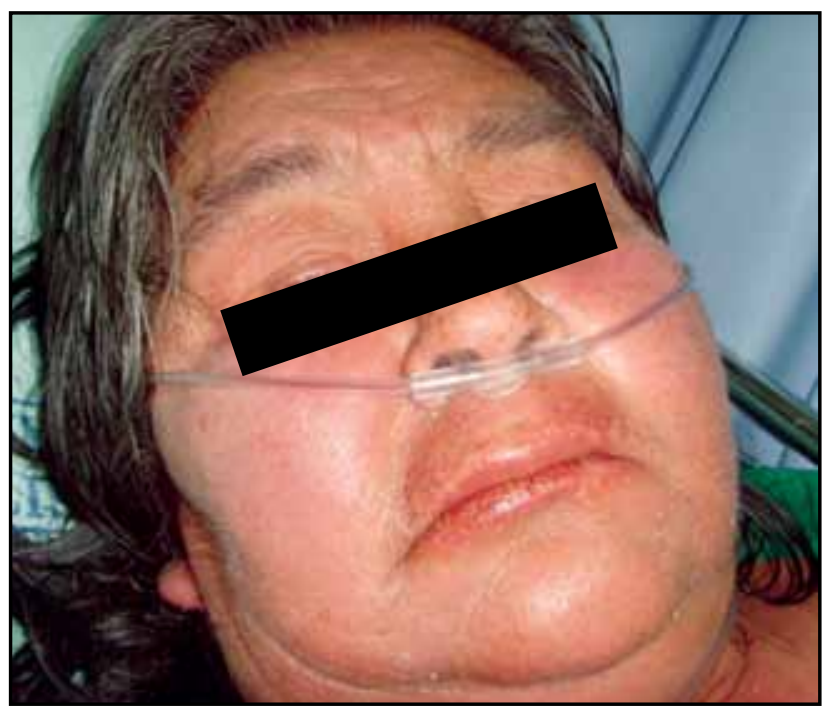

Figura 1D.

1) y las pruebas hepáticas evidenciaron una elevación de la transaminasas (Tabla 2).

Tres días después de su ingreso presentó hematoquezia y desarrolló herpes labial (Figura 1E). Se realizó un coproscópico sin evidencia de parásitos intestinales o proceso bacteriano y una colonoscopia donde se encontró hemorroides internas grado II congestivas y un pólipo en colón derecho. La biopsia demostró inflamación crónica severa, eosinofilia y cambio adenomatoso focal atípico. Se inició manejo con metilprednisolona y aciclovir oral y se continuó tratamiento con prednisolona $100 \mathrm{mg} /$ día (Figura 1 - Fotos A a D).

Se trata entonces de una paciente adulto mayor que cursa con reacción cutánea severa tipo hipersensibilidad con compromiso sistémico establecido por la presencia de eosinofilia y elevación de transaminasas quien además presentó durante su evolución reactivación de herpes labial. Por lo anterior se consideró diagnóstico definitivo de DRESS por sulfasalazina con score diagnóstico de cinco puntos. La 
Tabla 2. Química sanguínea.

\begin{tabular}{|c|c|c|c|c|c|c|}
\hline Fecha & $\begin{array}{c}\text { TGO } \\
(\mathbf{U} / \mathbf{L})\end{array}$ & $\begin{array}{c}\text { TGP } \\
(\mathbf{U} / \mathbf{L})\end{array}$ & $\begin{array}{c}\text { Bilirrubinas } \\
\text { totales } \\
(\mathbf{m g} / \mathbf{d L})\end{array}$ & $\begin{array}{c}\text { Bilirrubinas } \\
\text { directas } \\
(\mathbf{m g} / \mathbf{d L})\end{array}$ & $\begin{array}{c}\text { Bilirrubinas } \\
\text { indirectas } \\
(\mathbf{m g} / \mathbf{d L})\end{array}$ \\
\hline Diciembre 02/09 & 27 & 111 & 0.62 & 0.32 & 0.30 \\
\hline Diciembre 03/09 & 65 & 145 & & & \\
\hline Diciembre 06/09 & 76 & 157 & 0.6 & 0.31 & 0.63 \\
\hline
\end{tabular}

paciente presentó mejoría clínica con los esteroides y con el retiro de sulfasalazina.

\section{Reacciones cutáneas severas medicamentosas}

Las reacciones cutáneas severas son una de las manifestaciones adversas de los medicamentos que llegan a poner en peligro la vida. La fisiopatología no está bien dilucidada pero se presume la existencia de mecanismos inmunológicos, infecciosos y genéticos que en conjunto o por mecanismos independientes generan dicha respuesta.

\section{Factores inmunológicos}

La mayoría de reacciones severas a medicamentos son de hipersensibilidad retardada o tipo IV. Existen células tipo T específicas para medicamentos con potencial citotóxico y que orquestan la respuesta inflamatoria a través de liberación de diferentes citocinas y quimiocinas. La reacción tipo IVb (Th2) domina la presentación maculopapular con eosinofilia, y la respuesta CD8+ es la responsable de formación de bullas, necrolisis epidérmica tóxica y del compromiso sistémico (1); así mismo se describe la influencia de HLA en las reacciones cutáneas medicamentosas (2). Entre los otros mecanismos propuestos se encuentran las perforinas liberadas por células NK y linfocitos T CD8+ y la activación de receptores de membrana de activación de la apóptosis (como el Fas) (3).

\section{Factores infecciosos}

Existe interacción entre la respuesta inmune antiviral para herpes virus (EBV, HHV-6, HHV-7 y CMV) y la respuesta inmune específica para medicamentos. Hay reacción cruzada de células $T$ antivirales con el medicamento que desencadena respuesta inflamatoria con producción de anticuerpos contra los componentes del citocromo P450 (3). También se ha planteado una inducción de inmunosupresión de algunos medicamentos con la capacidad intrínseca de generar reactivación de virus latentes con respuesta sistémica inmune masiva, guiando a la pérdida de tolerancia inmune a otros medicamentos presentes en el organismo $(3,4)$.

\section{Factores genéticos}

La predisposición genética es un factor de riesgo para presentar hipersensibilidad a los medicamentos. Se ha men- cionado que existe un riesgo aumentado en individuos de raza negra y en pacientes con alteración en la producción y detoxificación de metabolitos activos, como la deficiencia heredada de la enzima peróxido hidrolasa que detoxifica intermediarios reactivos de algunos anticonvulsivantes; asî mismo los acetiladores lentos que producen metabolitos de hidroxiolamina tóxicos en las sulfonilureas también incrementan el riesgo (3-5). Este tipo de metabolito puede tener un efecto tóxico directo o actuar como hapteno alterando la tolerancia a proteínas endógenas.

\section{Otros}

Otro factor descrito es la disminución de niveles de cisteína y glutatión. La cisteína es el precursor del glutatión el cual es considerado el principal tiol intracelular que actúa como antioxidante (3).

\section{Síndrome de Steven Johnson y necrólisis epidérmica tóxica}

Actualmente se considera que el Síndrome de Steven Johnson (SSJ) y la necrólisis epidérmica tóxica (NET) constituyen un espectro de la misma enfermedad (6). Sin embargo, la prontitud en el diagnóstico es importante, ya que la mortalidad del SSJ es de aproximadamente 5\%, mientras que para la NET alcanza de 35\% a 40\% (7).

En la mayoría de los casos, el SSJ y la NET son inducidos por medicamentos. En el caso del SSJ los fármacos más comúnmente implicados son carbamazepina, barbitúricos, hidantoína, medios de contraste de yodohexol, oxifenilbutazona, penicilinas, penicilamina, fenobarbital, derivados de la fenotiazina, inhibidores de la transcriptasa inversa, salicilatos, sulfasalazina, sulfonamidas y derivados de la tetraciclina. Para la NET los agentes más frecuentemente descritos son alopurinol, antituberculosos, barbitúricos, carbamazepina, trimetropim sulfametoxazol, hidantoína, nitrofurantoína, AINEs, fenilbutazona, sulfonamidas y tetraciclinas (7).

\section{Presentación clínica}

Debido a que la mayoría de los casos son inducidos por medicamentos, los fármacos administrados dentro de 1-3 semanas son los más sospechosos; sin embargo, un tiempo de exposición mayor o menor no debe descartar un agente. En pacientes que toman medicamentos por largo tiempo, el mayor riesgo se encuentra en los primeros dos meses 
de uso. Hay casos descritos provocados por infecciones virales como HHV-6, HIV o bacterianas por Mycoplasma pneumoniae y Yersinnia (6).

El SSJ se caracteriza por una erupción cutánea generalizada compuesta por máculas parduscas y purpúricas, con tamaño y forma irregulares, que tienden a confluir, de predominio en tronco, signo de Nikolsky y afección de dos o más mucosas. El cuadro típico de la NET consiste en la aparición súbita, tras un pródromo "catarral" de lesiones eritematosas, dolorosas, inicialmente distribuidas de forma simétrica en cara y tronco, aunque posteriormente se pueden extender hacia las partes acras (Tabla 3). La evolución de estas lesiones es hacia la necrosis y el desprendimiento de la epidermis que produce ampollas flácidas y signo de Nikols$\mathrm{ky}$, con desprendimiento de amplias zonas epidérmicas, que dejan erosiones exudativas.

$\mathrm{El}$ área de superficie corporal afectada por las lesiones determina el diagnóstico ya que menos del 10\% indica SSJ, más de 30\% NET y entre 10-30\% se describe un cuadro de sobreposición de ambas presentaciones (8).

\section{Tratamiento}

La mayoría de los casos requiere manejo intrahospitalario. Los medicamentos sospechosos deben ser suspendidos inmediatamente. Se debe dar soporte nutricional, terapia de reposición hídrica, control de la sobreinfección de las lesiones y control de la temperatura. No se recomienda el uso de antibióticos profilácticos y su uso se reserva sólo cuando hay signos de infección o sepsis (7).

\section{Erupción fija generalizada bullosa medicamentosa}

Debe ser diferenciada del SSJ/NET. Existen parches violáceos cafés y ampollas flácidas. Las ampollas sólo afectan un pequeño porcentaje de la superficie celular corporal y entre las grandes ampollas siempre hay áreas de piel intacta. El compromiso mucoso es raro y cuando ocurre es leve. Los pacientes no se sienten enfermos ni con fiebre y estado general es mucho mejor que en los pacientes con STS/NET. La mayoría describen una historia de exantema local (erupción medicamentosa fija); sin embargo, la exposición repetida guía a un daño de la piel generalizado y a una enfermedad seria (5) (Tabla 3).

\section{Pustulosis exantematosa generalizada aguda}

Eritema extenso agudo con docenas de pústulas no foliculares especialmente en los pliegues y superficies flexoras. Raramente compromete mucosas y cuando lo hace los síntomas son moderados. Los pacientes tienen fiebre y neutrofilia (5) (Tabla 3).

\section{Criterios diagnósticos (5)}

Pústulas no foliculares, pequeñas, generalizadas, en un fondo eritematoso edematoso. Histología: pústulas intrae- pidermicas y/o subcorneales espongiformes e infiltrado perivascular con neutrófilos y edema de la dermis papilar. Puede haber vasculitis o queratinocitos necróticos. Fiebre > $38^{\circ} \mathrm{C}$. Neutrofilia. Inicio agudo. Resolución espontánea en menos de 15 días con descamación característica.

El diagnóstico diferencial se debe basar en descartar como primera medida desorden pustuloso infeccioso. La combinación de fiebre, neutrofilia y pustulosis es mal diagnosticado como infección severa aguda. Por eso debe tomarse biopsia para excluir otras enfermedades pústulosas cutáneas.

\section{Síndrome de DRESS}

El acrónimo DRESS (Drug Related Eosinophylia with Sistemic Symptoms o reacción a drogas con eosinofilia y síntomas sistémicos) fue propuesto en 1996 por Boquet y colaboradores, y describía un síndrome con múltiples manifestaciones sistémicas secundarias al uso de algunos medicamentos. El síndrome de DRESS es considerado ahora una toxicodermia medicamentosa de severidad importante y fisiopatología desconocida, en la que al parecer desempeñan un papel importante factores inmunológicos, virales e inflamatorios (9).

Diferentes grupos han tratado de establecer pautas diagnósticas, dentro de las cuales se destaca la propuesta por el grupo japonés $(1,9)$ (Tabla 3): erupción cutánea asociada con exposición medicamentosa. Fiebre $>38$. Hipertransaminasemia (elevación de transaminasas al menos dos veces de los valores normales). Alteraciones hematológicas: leucocitosis, eosinofilia $>1,500 / \mathrm{mm}^{3} \mathrm{y} / \mathrm{o}$ presencia de linfocitos atípicos (10). Adenopatías (> $2 \mathrm{~cm}$ de diámetro). Reactivación con virus herpes simple.

Otros grupos han incluido la nefritis intersticial y/o neumonitis intersticial y/o carditis, como variables en cuanto a compromiso sistémico y han dado especial importancia a la reactivación de HHV-6 (9).

\section{Epidemiología}

El síndrome de DRESS se presenta en todos los grupos poblacionales, siendo más prevalente en mujeres de raza negra y de severidad mayor en pacientes inmunocomprometidos. La incidencia de síndrome de DRESS con anticonvulsivantes es variable oscilando entre 1 por cada 1000 a 1 por cada 10000 expuestos (11). La mortalidad asociada es de $8-10 \%$ (3).

\section{Presentación clínica}

Se caracteriza por la aparición de fiebre y lesiones cutáneas eritematosas pruriginosas (pápulas, pústulas o vesículas) que comienzan en tronco y cara con posterior extensión al resto de segmentos corporales asociado a linfadenopatía, compromiso orgánico (hematológico, hepático, gastrointestinal, renal o miocárdico) (4) y edema periorbitario (5). Generalmente se presenta una a ocho semanas posterior a la exposición medicamentosa (3) y en algunos casos existe una fuerte asociación con la aparición de patologías 
virales (HHV-6, CMV, VIH). Se han documentado casos de compromiso pancreático, tiroideo y muscular asociado (3) (Tabla 3).

\section{Diagnóstico}

Requiere una adecuada historia clínica, asociación medicamentosa y aparición de clínica previamente descrita. Se deben realizar de rutina las siguientes pruebas de laboratorio: hemograma, función hepática y renal; y de acuerdo con la evolución y sospecha de compromiso orgánico se realizarán pruebas complementarias (función tiroidea, CK, ecocardiograma, colonoscopia, etc). La biopsia de piel aunque no es diagnóstica, sí puede ayudar a corroborar el diagnóstico.

Dentro del diagnóstico diferencial del síndrome de DRESS están: lupus eritematoso inducido por drogas, mononucleosis infecciosa, síndrome hipereosinofílico, enfermedad de Kawasaki, síndrome estafilocóccico del shock tóxico, Steven Johnsons (11).

\section{Tratamiento}

El tratamiento busca disminuir complicaciones, se indica manejo sintomático tópico, antihistamínicos y esteroides

\section{Discusión}

El desarrollo de DRESS por sulfasalazina no es común: a nivel mundial se han descrito alrededor de 10 casos en los últimos 10 años (Tabla 4). El nuestro representa un caso más en un paciente recibiendo sulfazalazina para el tratamiento de artritis reumatoidea. El diagnóstico se estableció con los hallazgos clínicos (erupción cutánea asociada a exposición mediamentosa, reactivación de herpes simple labial), paraclínicos (hipertransaminasemia, eosinofilia y leucocitosis) y de respuesta terapéutica (esteroides y suspensión de sulfasalazina con recuperación clínica). Se consideró diagnóstico definitivo al cumplir los criterios propuestos para diagnóstico de DRESS propuestos por Kardaun en el año 2007. Se asoció a sulfasalazina por

Tabla 3. Comparación de diferentes tipos de reacciones severas a medicamentos (Modificado de 5, 10).

\begin{tabular}{|c|c|c|c|c|}
\hline & $\begin{array}{c}\text { Síndrome de } \\
\text { Stevens-Johnson } \\
\text { y Necrolisis Epidérmica } \\
\text { Tóxica }\end{array}$ & $\begin{array}{c}\text { Erupción fija } \\
\text { generalizada } \\
\text { bullosa } \\
\text { medicamentosa }\end{array}$ & $\begin{array}{c}\text { Pustulosis } \\
\text { exantematosa } \\
\text { generalizada } \\
\text { aguda }\end{array}$ & $\begin{array}{c}\text { Síndrome } \\
\text { de hipersensibilidad } \\
\text { o DRESS }\end{array}$ \\
\hline Latencia después de tomar medicamento & 1-3 Semanas & 1 Semana & Pocos días & 2-6 semanas \\
\hline Duración de la reacción & 1-3 Semanas & 1-2 Semanas & 1 Semana & Muchas semanas \\
\hline \multicolumn{5}{|c|}{ Hallazgos clínicos } \\
\hline Fiebre & +++ & + & +++ & +++ \\
\hline Edema facial & - & - & ++ & +++ \\
\hline Pústulas & - & - & +++ & + \\
\hline Pápulas infiltradas & - & - & ++ & +++ \\
\hline Ampollas & +++ & +++ & + (Tesión) & $+($ Tesión $)$ \\
\hline Lesiones Diana & +++ & (Máculas largas) & $+1-$ & $+/-$ \\
\hline Compromiso de mucosa & +++ & $+1-$ & $+1-$ & Queilitis \\
\hline \multicolumn{5}{|c|}{ Hallazgos paraclínicos } \\
\hline Neutrofilia & $\downarrow$ & $\downarrow$ & $\uparrow \uparrow \uparrow$ & $\uparrow$ \\
\hline Eosinofilia & - & - & $\uparrow$ & $\uparrow \uparrow \uparrow$ \\
\hline Linfocitos atípicos & - & - & - & + \\
\hline Compromiso de otros órganos & $\begin{array}{c}\text { Necrosis traqueobronquial } \\
\text { Nefritis tubular } \\
\text { Hepatitis }\end{array}$ & & $\begin{array}{c}\text { Hepatitis } \\
\text { Linfadenopatia }\end{array}$ & $\begin{array}{c}\text { Neumonía intersticial } \\
\text { Nefritis intersticial } \\
\text { Hepatitis, Carditis } \\
\text { Linfadenopatia }\end{array}$ \\
\hline Cambios histológicos en piel & Necrosis epidérmica & Necrosis epidérmica & Pústulas subcorneales & Infiltrado linfocítico \\
\hline Medicamentos implicados & $\begin{array}{c}\text { Anticonvulsivantes } \\
\text { aromaticos, lamotrigina, } \\
\text { antibióticos sulfaminados, } \\
\text { alopurinol, piroxicam, dapsona, } \\
\text { nevirapina }\end{array}$ & & $\begin{array}{c}\text { Betabloqueadores, } \\
\text { macrolidos, } \\
\text { calcioantagonistas, } \\
\text { aminopenicilinas, quinolonas, } \\
\text { antimalaricos }\end{array}$ & $\begin{array}{l}\text { Anticonvulsivantes aromáticos, } \\
\text { lamotrigina, alopurinol, } \\
\text { minociclina, dapsona, } \\
\text { sulfazalasina, metimazol, } \\
\text { talidomida. }\end{array}$ \\
\hline Tratamiento & IgIV, Ciclosporina, Soporte & & $\begin{array}{c}\text { Sintomático } \\
\text { Esteroides sistémicos }\end{array}$ & Esteroides sitmémicos \\
\hline
\end{tabular}


Tabla 4. Score diagnóstico de DRESS (Kardaun et al 2007).

\begin{tabular}{|c|c|c|c|c|c|c|}
\hline SCORE & -1 & $\mathbf{0}$ & 1 & 2 & MIN & MAX \\
\hline Fiebre & No & $\mathrm{Si}$ & & & -1 & 0 \\
\hline Adenopatías & & No & $\mathrm{Si}$ & & 0 & 1 \\
\hline Eosinofilia & & & $700-1499 u / 1$ & Mayor $1500 \mathrm{u} / \mathrm{l}$ & 0 & 2 \\
\hline Linfocitos atípicos & & No & SI & & 0 & 1 \\
\hline Compromiso piel & & No & MAS DE $50 \%$ & & -2 & 2 \\
\hline $\begin{array}{l}\text { Compromiso de órganos } \\
\text { Hígado } \\
\text { Riñón } \\
\text { Pulmón } \\
\text { Corazón } \\
\text { Páncreas } \\
\text { Otros órganos }\end{array}$ & & & No & $\begin{array}{l}\mathrm{Si} \\
\mathrm{Si} \\
\mathrm{Si} \\
\mathrm{Si} \\
\mathrm{Si} \\
\mathrm{Si} \\
\mathrm{Si}\end{array}$ & 0 & 2 \\
\hline Resolución $>15$ días & No & $\mathrm{Si}$ & & & -1 & 0 \\
\hline $\begin{array}{l}\text { Serología-PCR } \\
\text { Hepatitis } \\
\text { VEB } \\
\text { CMV } \\
\text { Mycoplasma } \\
\text { Chlamydia }\end{array}$ & & & $\mathrm{Si}$ & & 0 & 1 \\
\hline TOTAL & & & & & -4 & 9 \\
\hline
\end{tabular}

la exposición al medicamento las dos semanas previas a las manifestaciones clínicas y se descartó que la reacción fuera secundaria a metotrexate, naproxeno, calcio y omeprazol por su uso rutinario desde hace dos años sin ninguna complicación.

Otro punto importante por tratar en esta revisión es la colitis eosinofílica, una entidad de etiología multifactorial, frecuente en niños y adultos jóvenes (30-50 años de edad). El 50-90\% de los casos tienen eosinofilia periférica $\mathrm{y}$ antecedente de alergia o reacciones de hipersensibilidad en un porcentaje mayor a $30 \%$. El principal criterio de diagnóstico histopatológico es la presencia de infiltrados eosinofílicos y la presentación clínica y el compromiso sistémico son variables dependiendo del estado de salud previo y las comorbilidades asociadas. El diagnóstico diferencial de colitis eosinofílica debe hacerse con colitis parasitaria (Enterobius vermicularis, Strongyloides stercoralis y Trichuris trichiura), colitis inducida por medicamentos (clozapina, carbamazepina, AINES y rifampicina), síndrome hiperesonofílico, enfermedad inflamatoria intestinal. Enfermedades del tejido conectivo y síndrome de Tolosa Hunt, entre otros (12).

\section{Referencias}

1. Wolf R, Matz H, Marcos B, Orion E. Drug rash with eosinophilia and systemic symptoms vs toxic epidermal necrolysis: the dilemma of classification. Clin Dermatol 2005; 23(3): 311-4.

2. Wolff K LAG, Katz SI, Gilchrest BA, Paller AS, Leffell DJ. Fitzpatrick's Dermatology in General Medicine 2008; 325-328.

3. Seth D, Kamat D, Montejo J. DRESS syndrome: a practical approach for primary care practitioners. Clin Pediatr (Phila) 2008; 47(9): 947-52.

4. Michel F, Navellou JC, Ferraud D, Toussirot E, Wendling D. DRESS syndrome in a patient on sulfasalazine for rheumatoid arthritis. Joint Bone Spine 2005; 72(1): $82-5$.

5. Mockenhaupt M. Severe drug-induced skin reactions: clinical pattern, diagnostics and therapy. J Dtsch Dermatol Ges 2009; 7(2): 142-60.

6. Doval IG. Necrólisis epidérmica tóxica y síndrome de Stevens-Johnson: clasificación y actualidad terapéutica. Actas Dermosifiliogr 2000 ; 91: 541-51.

7. Crowson AN, Brown TJ, Magro CM. Progress in the understanding of the pathology and pathogenesis of cutaneous drug eruptions : implications for management. Am J Clin Dermatol 2003; 4(6): 407-28.

8. Taylor CR, Gandhi RT, Handwerker J, Duncan LM. Case records of the Massachusetts General Hospital. Case 34-2009. A 20-year-old man with sore throat, fever, and rash. N Engl J Med 2009; 361(18): 1787-96.

9. Shiohara T, Lijima M, Ikezawa $\mathbf{Z}$, Hashimoto $\mathbf{K}$. The diagnosis of a DRESS syndrome has been sufficiently established on the basis of typical clinical features and viral reactivations. Br J Dermatol 2007;156(5): 1083-4.

10. Bachot N, Roujeau JC. Differential diagnosis of severe cutaneous drug eruptions. Am J Clin Dermatol 2003; 4(8): 561-72.

11. Kano Y, Shiohara T. The variable clinical picture of drug-induced hypersensitivity syndrome/drug rash with eosinophilia and systemic symptoms in relation to the eliciting drug. Immunol Allergy Clin North Am 2009; 29(3): 481-501.

12. Okpara N, Aswad B, Baffy G. Eosinophilic colitis. World J Gastroenterol 2009 15(24): 2975-2979 